\title{
Privacy Unraveling Around Explicit HIV Status Disclosure Fields in the Online Geosocial Hookup App Grindr
}

\author{
MARK WARNER, University College London, UK \\ ANDREAS GUTMANN*, OneSpan, UK \\ M. ANGELA SASSE, University College London, UK \\ ANN BLANDFORD, University College London, UK
}

\begin{abstract}
mHealth applications ("apps") must be searched for and downloaded prior to use, creating a potential barrier to uptake. Integrating health interventions into existing online social environments removes this barrier. However, little is known about the effects of linking sensitive health information to existing online identities. Our qualitative analysis of online comments $(\mathrm{n}=192)$ explores the user views of an HIV intervention integrated into the geosocial hookup app Grindr. We find some HIV positive users report keeping their status private to reduce their stigma exposure, whilst others report publicly disclosing their status to avoid being stigmatised by others. Where users keep their status private, we find concerns that social assumptions may develop around these non-disclosures, creating a privacy unraveling effect which restricts disclosure choice. Using Peppet's four proposed limits to privacy unraveling, we develop a set of descriptive conceptual designs to explore the privacy respecting potential of these limits within this context and propose further research to address this privacy challenge.
\end{abstract}

CCS Concepts: • Human-centered computing $\rightarrow$ Empirical studies in collaborative and social computing; Social networks; • Security and privacy $\rightarrow$ Social aspects of security and privacy;

Additional Key Words and Phrases: Privacy Unraveling; Privacy; Self-disclosure; Identity; HIV Disclosure

ACM Reference Format:

Mark Warner, Andreas Gutmann, M. Angela Sasse, and Ann Blandford. 2018. Privacy Unraveling Around Explicit HIV Status Disclosure Fields in the Online Geosocial Hookup App Grindr. Proceedings of the ACM on Human-Computer Interaction 2, CSCW (November 2018), 22 pages. https://doi.org/10.1145/3274450

\section{INTRODUCTION}

Human Immunodeficiency Virus (HIV) interventions have been developed as stand-alone apps to increase awareness, reduce risk, and promote safer sex and regular testing [58]. Yet, they have attracted little user attention and few positive reviews [16, 46]. For health intervention apps to be effective, people must have intent to change by actively searching for and downloading the app, and then continue to engage with it. Reducing this barrier, a number of geosocial hookup apps have integrated HIV interventions into their platforms. These provide users with the option to publicly disclose their HIV status to other users. However, little is known about how introducing sensitive health data into these environments affects user privacy. This was evident when security researchers recently identified that Grindr was sharing its users' personally identifiable HIV status information with third-party companies [26].

In this paper, we explore whether these disclosure fields support marginalised groups who may feel unable to publicly disclose their status, especially in the early stages of accepting the

*Also with University College London.

Authors' addresses: Mark Warner, University College London, 66-72 Gower Street, London, WC1E 6EA, UK, mark.warner@ ucl.ac.uk; Andreas Gutmann, OneSpan, Cambridge, UK, andreas.gutmann@onespan.com; M. Angela Sasse, University College London, Malet Place, London, UK, a.sasse@ucl.ac.uk; Ann Blandford, University College London, 66-72 Gower Street, London, UK, a.blandford@ucl.ac.uk. 
diagnosis [7] where people require support and positive interactions [62]. Around half of MSM who live with HIV in the UK reported feeling of shame, guilt, and low self-esteem in relation to their HIV status in the 12 months post diagnosis [60]. Systems should be designed in a way that neither disadvantages nor stigmatises any group of users [9]. Therefore, these marginalised users should be in control over when they choose to disclose potentially stigmatising personal health information. We report on a study in which we collected and analysed the online user views of introducing a structured field for publicly disclosing HIV status information in the popular geosocial hookup app Grindr, used by men who have sex with men (MSM).

Throughout this paper, we refer to the HIV disclosure option as an HIV intervention. The intended outcome of this intervention as reported by Grindr is to "create an open dialogue among our users about sexual health" [17]. Building Healthy Online Communities (BHOC) $)^{1}$, who have advised Grindr on issues related to sexual health, stated that this intervention would also help create "a healthy online community through supporting HIV prevention and fighting stigma" [17]. This explicit disclosure field is part of a wider health intervention by Grindr, and since conducting this study, Grindr have also introduced testing reminders at certain intervals for users who choose to use this option [16].

With online geosocial dating apps expanding the means by which MSM are meeting, Grindr (released in 2009) has become the most popular of these apps within the MSM community. It has more than 3 million daily active users worldwide, who spend an average of 54 minutes interacting with the app each day [31]. App usage is primarily for finding hookups [61], but users also report usage for socialising, friendship, entertainment, dating, and gay community involvement [53, 64]. In contrast to interventions which focus on promoting awareness, Grindr proposed implementing an explicit field for publicly reporting HIV status on user profiles, and the ability to filter users based on their status. In 2016, Grindr conducted a survey related to these proposals. Whilst the results of the survey have not been published, it acted as a catalyst for online discussion. Several online websites attracted user comments related to the proposed disclosure of and filtering by HIV status.

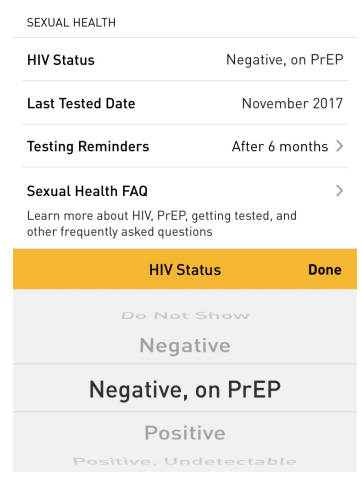

Fig. 1. Cropped screenshot of the explicit HIV status disclosure field in Grindr.

Grindr later modified its app to include an explicit field for reporting HIV status and last tested date information as shown in Figure 1. The implementation of this feature also attracted online comments and discussion. As HIV filtering was never implemented, our analysis and discussion

\footnotetext{
${ }^{1} \mathrm{BHOC}$ are a consortium made up of public health leaders and key individuals from the gay dating industry who are working together support HIV and STI prevention online.
} 
is focused on the HIV status and last test date disclosure fields. Where we mention the public disclosure of HIV status, 'public' refers to a user's profile space within Grindr. Whilst not truly public as to view this information requires a Grindr account, we refer to it as being public within the Grindr environment. Our paper makes several contributions to the privacy and social computing literature.

- Our empirical analysis of online comments provides a first look at user perceptions of explicit HIV status disclosure field in an online hookup app used by MSM.

- We identify privacy unraveling [50] concerns around these explicit disclosure fields. Whilst Peppet's [50] privacy unraveling effect has been observed in economic environments, to the best of our knowledge this is the first time it has been explored in an online social environment.

- Finally, we build on Peppet's work by developing a number of sub-categories and descriptive conceptual designs as a way of reducing the unraveling effect. These conceptual designs are developed to address the privacy concerns of marginalised groups who are most at risk of stigma as a consequence of this effect. In developing these designs, we provide a first look at how these limits could be applied in design, and extend the literature on privacy unraveling, identifying a new application domain within online social environments.

\section{BACKGROUND AND RELATED WORK}

This section provides an overview of the background and theories used to understand our empirical findings. We start by providing a brief overview of the HIV virus and treatments in the UK. We then discuss several social interaction theories in relation to HIV disclosure. As identity plays an important role in online social interaction, we present a brief overview of identity management and the role privacy plays in supporting both online social interactions and identity management. Finally, we explore privacy unraveling, providing a detailed definition and an overview of previous research which has explored its effects.

\subsection{Human Immunodeficiency Virus (HIV)}

In the UK, $54 \%$ of those newly diagnosed with HIV in 2016 identified as MSM, of which $32 \%$ were late diagnoses [13]. The majority of HIV transmissions in the UK are through sexual contact [23]. MSM living with HIV but unaware of their status was estimated to be $13 \%$ of the total number affected [13]. A high proportion of new infections amongst MSM are caused by HIV positive individuals unaware of their status [10,32, 51]. People living with HIV in the UK who are treated with highly active antiretroviral therapy (HAART) often describe their status as being "undetectable". A term used to describe individuals who are HIV positive, but are responding well to treatment and have an undetectable level of the virus in their blood (and are therefore no longer at risk of forward transmission $[54,55])$.

Serodiscordant is a term used to describe a sexual partnership where one individual has tested positive for HIV, and the other is HIV negative. Studies show HAART to be effective at minimising transmission between serodiscordant heterosexual couples [5,21], as well as between serodiscordant MSM engaged in unprotected anal intercourse (UAI) [54, 55]. This has led to reduced transmission rates by those aware of their status and being treated, whilst those unaware continue to be a risk. In recent years, new HIV prevention drugs, pre-exposure prophylaxis (PrEP), have been made available to individuals at risk of HIV infection. These medical interventions have created an array of HIV status options that people may be identified as, or self-identify as, summarised in Figure 2. Grindr provides users with five disclosure options: Do Not Show, Negative, Negative on PrEP, 
Positive, and Positive Undetectable. Below we briefly discuss what these options mean and who may self-identify with them.

If an individual has tested negative for HIV, they may identify as being negative; however, those untested may also identify as negative in the absence of a status unknown option. Of those who test negative, prevention drugs can be used to avoid HIV on exposure. These individuals may self-identify as being negative on PrEP and are typically required to test for HIV on a regular basis (6 months). There are two ways in which PrEP can be taken, daily (one tablet per day), or event based dosing (a number of tablets prior to and after sex), but the disclosure options in Grindr do not allow for this distinction. For those diagnosed as HIV positive, the effectiveness of modern treatments means that becoming undetectable and untransmittable is often achieved soon after diagnosis. If tests show an undetectable viral load for $>6$ months, guidance within the UK states that they are then classed as being undetectable [48] and may then self-identify as being positive undetectable. If an individual responds badly to medication, or fails to adhere to medication, they may self-identify as positive. In both cases, in the UK, regular testing is recommended to monitor the status of the virus.

\subsection{Self-Disclosure of HIV Status Information}

On initial interaction, people have self-reported that to maximise their social desirability within their social environment, they self-disclose at a superficial level and gradually increase in intimacy and breadth as trust develops [66]. Whilst self-disclosure is important for relationship development, it can also increase a person's vulnerability to negative social consequences. In this paper we use the terms 'self-disclosure' to mean any form of intentional disclosure of HIV status [4], and 'serodiscussion' to refer more specifically to conversation based disclosures. Serodiscussion within couples can help individuals make more informed sexual risk decisions for themselves and their partners [68]. Serodiscussion not only informs, it also allows for the exchange of richer information that can educate. Educating others can act as an incentive for both men and women to disclose their HIV status [20], helping to change the out-of-date views and discourse that HIV still attracts [47].

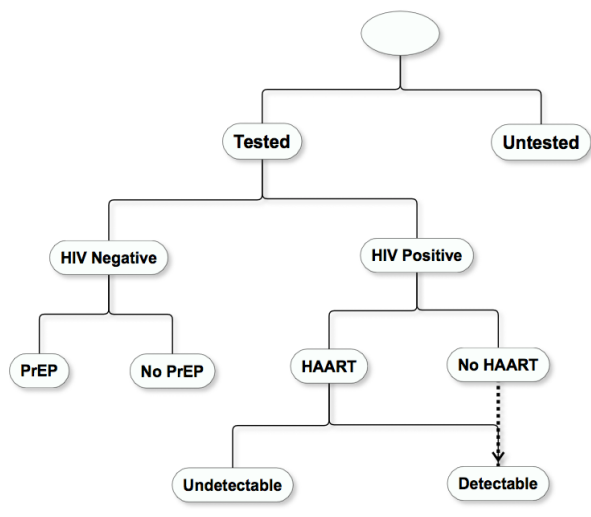

Fig. 2. Tree diagram showing a summary of HIV status conditions.

Self-disclosing HIV status helps to create more openness, reducing stigma through the normalising of disclosure within society [15]. Paradoxically, however, for many MSM the attached stigma 
and subsequent fear of rejection creates a barrier to disclosure during sexual negotiations $[19,68]$. Derlega et al.'s [20] study shows that fear of rejection, privacy, and self-blame can result in the non-disclosure of HIV status information within certain close interpersonal relationships. The factor of privacy is often linked with an individual's need to protect themselves from HIV related stigma $[1,30,56]$. MSM may avoid serodiscussion through fear of an exciting sexual experience becoming too serious, hampering the sexual mood of the interaction and being an unwanted reminder of their condition [14]. In a study of MSMs' online sexual negotiations, HIV negative men were found to be more likely to self-disclose their status than HIV positive men, with a large proportion of the latter reporting to have misreported their status [14]. Prior to Grindr implementing an explicit field for HIV disclosure, only a small number of users reported their HIV status on their Grindr profile using terms like "ddf" ("drug and disease free") [11].

\subsection{HIV Threat to Identity}

Users of social apps self-present by engaging in "profile work", exerting effort to maintain and manage their online personas [57]. Users may act to promote their profile, actively emphasising some aspects of their identity whilst concealing others with the goal of appearing desirable to their audience within a given social environment [45]. On being diagnosed with HIV, MSM may change their behaviour whilst going through identity transition, a term used to describe a multi-stage process of incorporating a new element into their identity [62]. They can find it difficult to integrate the illness as part of their self-construal, especially during early stages of this transition [25]. Identity transition can be aided through positive self-disclosures and interactions within social groups, helping individuals achieve a sense of belonging and maintain self-esteem [7, 62].

Being HIV positive is often seen as an undesirable attribute [62]. Those fearful or uncertain of how this new aspect of their identity will be perceived by others may act to more closely regulate self-disclosures and, thus, minimise the risk of a negative social response. Similarly, as the preventative drug PrEP becomes more widely used, research has found it attracting its own stigma, with impressions developing around PrEP users as being more promiscuous and into higher risk sex $[29,38]$. It is therefore important to understand the effect of introducing HIV status information into an existing online identity, and to evaluate how disclosure is managed to ensure users maintain disclosure choice so they can effectively regulate self-disclosures in accordance with their needs.

\subsection{Privacy in Social Interactions}

Control of personal information, and how it is perceived by others, is an important aspect of identity management and often described when the concept of privacy is being explored [39, 41]. Understanding privacy as a user-centric, contextualised concept can help explain variations in privacy concerns. The Adams and Sasse privacy model [2] suggests people evaluate privacy using three interrelated factors. They judge the sensitivity of the information being disclosed, evaluate the trust in the information receiver and assess the cost/benefits of how the information is to be used. However, this model assumes that the initial disclosure is an explicit choice made by the user. In social interactions, people view information as having varying degrees of sensitivity and social value. In a dating environment, where an attribute - like an HIV negative status - increases attractiveness or desirability to others, it is then in the best interest for users with that attractive attribute to disclose. This can create a social privacy problem known as the privacy unraveling effect [50], limiting the effectiveness of control as a mechanism for respecting privacy. We use the term 'social privacy' as opposed to 'individual privacy' or just 'privacy' to recognise the effect the social environment has on the manifestation of this phenomenon. 


\subsection{Privacy Unraveling}

Peppet [50] proposes unraveling as a privacy threat in a "full disclosure future" where it becomes expected that personal information is "signalled" to others in order for them to be distinguishable from others. For example, where a driver wants to signal their reduced risk to their insurer, they may allow a device to track their speed, cornering, and braking; where a life insurance customer wants to signal their healthy lifestyle, they may use a health tracker wearable to send daily step-counts, heart rate and sleep quality information. Privacy may unravel around those who choose to withhold information, as others may assume them to be "hiding" undesirable information, and could lead to them being stigmatised or penalised as a consequence [50].

This effect has been observed in an empirical study involving the disclosure of productivity costs within the labour market [8] and discussed in relation to the selling of cars on the online market place eBay [43]. Our search of the literature has only identified reports of this effect occurring within economic markets where goods and services are exchanged, but this effect may also exist in online social environments where individuals enact full disclosure to signal their social desirability to others.

To limit the effect of privacy unraveling, Peppet [50] suggest four mechanisms: transaction cost, unverifiability of ignorance, inability to accurately infer the negative, and norms. Negative assumptions may develop around non-disclosures when disclosing a desirable attribute is low-cost, as disclosure can be perceived as being an "obvious choice" for those with a desirable attribute. The first limitation suggests that if the cost of disclosing is increased, the "obvious choice" becomes less obvious, reducing stigmatising signals from non-disclosures. The second limitation proposed is unverifiability of ignorance. This limitation occurs when it is not possible to verify whether the disclosing party is aware of the state of the attribute not being disclosed. Peppet [50] uses the example of a transaction of a crate of oranges. Assuming the buyer is unable to verify that the seller knows how many oranges are inside the crate, if the seller does not disclose, the buyer is unable to draw negative inferences from non-disclosure due to the uncertainty over the seller's ignorance. The third limitation occurs when an inability exists that inhibits negative inferences being accurately inferred around non-disclosure. If the receiver of the signalled information is unable to comprehend that information, it will be difficult for them to develop assumptions from non-disclosures. Lastly, where norms develop around non-disclosure of information or actions, negative assumptions are much less likely to develop. An example of this can be seen in Germany, where it is much more common for home owners to request that their homes be blurred out on Google Street View to respect their privacy. In the UK, blurring of a home is seen as unusual, and may signal that they have something to hide, whilst in Germany, the norms around the use of this feature limit this privacy unraveling effect.

\section{METHODOLOGY}

The purpose of this study was to identify user views of introducing HIV status information into Grindr, the online geosocial hookup app used by MSM. We were interested in understanding the impact this may have on user privacy due to the sensitivities and stigma associated with HIV, and because this information would be associated with an existing online social identity. We examined the online views of users before and after Grindr implemented this feature into its app in November 2016 [17].

\subsection{Use of Online Comments}

Online comments have previously been used to understand public health views [27, 40], to study public discussion [24, 65], and in HCI research to develop design recommendations [59]. Conducting 
research with stigmatised populations that are often hard to reach has led researchers in the social sciences to utilise online platforms and data sources [44]. These resources include user-generated comments in naturalistic settings such as forums and in the comments section on news websites. The user-driven nature of these comments may be well suited to revealing the issues that matter most to the individuals posting [36]. We recognise that the use of online comments has limitations; these are discussed in section 6.

\subsection{Data Collection}

When conducting our online searches we used an anonymous browser to reduce the risk of customised search results being returned. Searches to identify news articles and blog posts reporting on the Grindr survey or the introduction of HIV reporting within Grindr were conducted with Google Search and DuckDuckGo using multiple keywords ${ }^{2}$. We found 29 websites related to this interface change. As filtering by HIV status was never implemented, our research focused on the disclosure of HIV status within the app. Therefore our criteria for inclusion were (1) the article or blog post was primarily about either the survey conducted by Grindr, or the later introduction of HIV status information in Grindr, and (2) at least one user comment had been posted. Using these criteria, 13 of the 29 websites found were selected. These websites containing a total of 149 comments posted between July 2016 and August 2017. These were added to a corpus of News Website (NW) comments. As a secondary source of data, we searched the UserVoice.com product feedback website to find user comments related to HIV disclosure within Grindr using the keyword "HIV". UserVoice.com is a managed customer feedback service used by Grindr to enable users to submit feedback and suggestions, and for other users to comment on that feedback. Our inclusion criteria for this source were (1) the user comment was primarily about HIV disclosure, and (2) the comment was related specifically to the Grindr application. This search identified a further 43 comments posted between November 2016 and May 2017. These were added to a corpus of Product Feedback (PF) comments. Figure 3 shows the word count and distribution of comments collected, separated by source, and indicates a good distribution of comments across the 14 website sources that matched our inclusion criteria. The mean length of all the comments was 87 words. The largest comment consisted of 1134 words, and the shortest was 6 words.

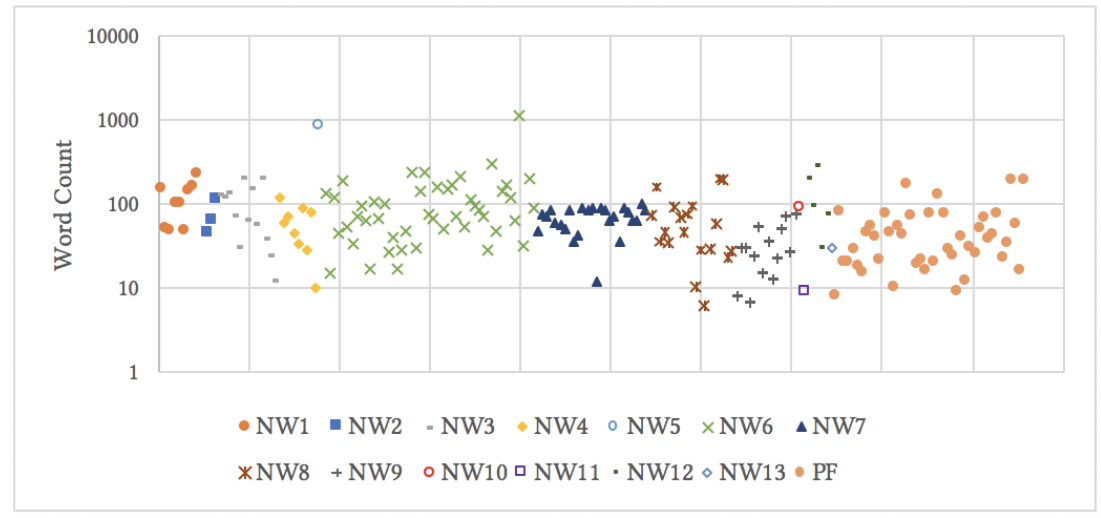

Fig. 3. Scatter plot of word count distribution ( $y$-axis, log transformed) of all user comments ( $x$-axis) separated by News Website (NW) and Product Feedback (PF) source websites.

${ }^{2}$ HIV Disclosure Grindr, HIV Filter Grindr, HIV Filter Grindr survey 


\subsection{Comment Analysis}

Using a thematic analysis [12], the first author systematically reviewed each comment and coded them iteratively in NVivo 11. This allowed the analyst to become familiar with the data and to understand it within the context in which it had been written. On completion of the coding, the codes were grouped into themes and reviewed and revised by the second author.

Supplementary analysis was performed to identify whether particular views were more prominent for a particular HIV status group; we analysed the data for people explicitly disclosing their HIV status. We identified $39(20.31 \%)$ comments which contained an explicit HIV disclosure. Of these, $32(16.67 \%)$ reported a HIV positive status and 7 (3.65\%) a HIV negative status, whilst 153 (79.69\%) did not disclose. No comments were found containing explicit unknown status disclosures. Some of the comments in the corpus contained implied status disclosures; however, as they were not explicit and were subject to interpretation, they were grouped with non-disclosure for these statistics.

We also wanted to understand whether the sources (news articles/blog posts) and corpus of comments were biased towards people holding positive, negative or neutral views towards the intervention. To determine this, two of the authors conducted a manual sentiment analysis of both the sources and the comments. They independently labelled them as positive, negative, or neutral towards the intervention. Both raters met in person, discussed cases of disagreement between their ratings, and found additional cases of agreement, e.g. cases that were "borderline" or "on the fence" between neutral and positive or negative. The values reported below are the averages of the two raters (e.g. "average 2.5 were positive"). The Cohen's (unweighted) kappa $\kappa$ was calculated across all source websites, and separately across the corpus of comments. Of the 13 source websites matching our inclusion criteria, 2.5 were positive, 6 were negative, and 4.5 were neutral $(\kappa=0.87)$. Of the comments, $68.5(35.68 \%)$ were marked positive, 61.5 (32.03\%) negative, and $62(32.29 \%)$ neutral $(\kappa=0.85)$. This shows a good sentiment distribution of sources and comments, and a strong rate of agreement between raters. No additional quantitative analysis has been performed on this data, so these views are not necessarily generalisable to all app users. Quotes from the initial web searches are referenced using the abbreviation ' $\mathrm{NW}$ ', followed by the website source number. Comments from the managed product feedback website are referenced using the abbreviation 'PF'.

\subsection{Ethics of Using Online Data}

This study has been approved by University College London ethics committee (ref: 11699/003). We requested that this study be reviewed for ethical approval due to the sensitive nature of some of the comments we were proposing to collect. During the process of the ethics review we consulted with colleague to deliberate the ethical issues around the use of this data. Through these deliberations, the primary concern we identified was the anonymity of the original data subjects when reporting our findings due to the sensitivities around the information. This was also a concern as it was not possible to obtain informed consent from participants as users had posted anonymously or pseudonymously, or no realistic mechanism was available to make contact with them. Whilst the original data subjects posted in an online public space for the purpose of public consumption, an implied audience and purpose limitation existed that our research would otherwise extend. We became aware that, even if we were to publish direct quotes anonymously, these could be re-identified using an online search engine. As a result, where an original comment contained a user's real name or a username which could be easily re-identified, we either opted not to reproduce the comment in this paper or paraphrased the comment. In circumstances where paraphrasing is used, consensus on the wording was gained from at least three of the named authors to ensure accurate representation of the original content. We believe the steps we have taken mitigate any 
risk to the original data subjects whilst maintaining valuable insight into the views of an often hard to reach population.

\section{FINDINGS}

We identified a number of themes related to the attitudes and concerns of Grindr users around the use of their HIV status information. In this paper, we focus on the four most distinct themes: managing of sexual health, desire to reduce exposure to stigma, trust of other users, and attitudes and concerns around disclosure choice.

\subsection{Managing Sexual Health}

The introduction of an explicit HIV status disclosure field in Grindr was intended to create a more open environment around sexual health information, allowing users to better manage their own sexual health [17]. Concerns around users' sexual health were a regular point of discussion in the analysed comments, with safe sex practices and education and awareness being of key concern. Yet, as people's opinions differed, a variety of sub-themes emerged. Increasing awareness and educating people within Grindr was raised in a number of comments, and this appeared especially important to undetectable users. As the undetectable status option was relatively new in the context of HIV, a lot of education within the community was still needed. Without this increased awareness and improved education, those with this status may find themselves having to regularly explain, and in some cases convince people of, the validity of their status and its sexual health consequences.

Appearing as undetectable will help the minds to understand better what it means. Removing the status option would send back hiv + guys to darkness. I appear on grindr as undetectable and I have discussion with others who are not aware about it so it helps to make minds improve (Original comment from PF)

Having discussions about HIV status information to educate and raise awareness was often mentioned in the comments, with some preferring to have a private discussion with other users rather than publicly disclosing their status on their profile.

People should always ask before having sex, it doesn't need to be posted for Everyone [sic] to see (Original comment from PF)

Whilst there were some users who felt that public disclosure could help stimulate discussion, others felt it would limit these discussions as the information was available on a user's profile, and therefore no longer needed to be discussed. With the introduction of the preventative drug PrEP and the increased awareness around the undetectable status, some felt that disclosure would be much less important in the future, as PrEP can prevent transmission, whilst effective HIV treatment can significantly reduce the risk of forward transmission. However, others were keen to point out that HIV was not the only STI of concern, with viruses like gonorrhea and hepatitis being a risk when engaging in unprotected anal intercourse.

I hope the guy with Hep B discloses! It's much more infectious than HIV, and can kill you just as dead. It'd be real ironic if for all his sanctimony about disclosure and victimhood, he was out there passing around his disease to unsuspecting victims (Original comment from NW1)

Whilst some felt HIV status information being made public could help them make better sexual risk decisions, others felt it could encourage people to have more unsafe sex. To counter this, some users suggested taking a default assumed state over other people's HIV status, assuming they were positive to reduce their risk of infection. 
HIV status is completely stupid. You should always assume that a new partner might be positive and practice safe sex. Saying someone is negative just encourages unsafe sex practices (Original comment from $\mathrm{PF}$ )

Clearly sexual health is an important issue for some Grindr users, especially as the application is predominately used for finding sexual hookups [61]. However, there were conflicting views around introducing HIV status information into Grindr, with some seeing it as a way of increasing awareness and reducing risk, whilst others held multiple contrasting views which we explore below.

\subsection{Managing Stigma}

Stigma was a significant theme that emerged from our analysis. Previous research finds that stigma can create a barrier to disclosure during sexual negotiations due to concerns of social exclusion and loss of sexual opportunity [47]. Our study supports these findings, but in addition we also find comments suggesting stigma could act to motivate disclosure for some users, as it could provide a way for them to reduce their stigma exposure. We found considerable concern related to the public disclosure of HIV status information on user profiles due to the stigma attached to HIV. There was concern that public disclosures would increase stigma rather than reduce it, and that it would disproportionally affect the subset of HIV positive users.

In my opinion it is awful to make people expose part of their medical record. This is sensiteve [sic], highly personal info. What is next? A full list of STDs check list? In many countries HIV comes with stigma so the only result of having this option on the app is to make most people lie about their status. It's dicsreminating [sic], racist even. I am not $\mathrm{HIV+}$ but will soon delete Grindr if this goes as it is now. (Original comment from PF)

Supporting previous research, we found comments suggesting reduced sexual opportunity may be more likely if HIV status information was made public, with concerns that people would be stereotyped and rejected based on their perceived stereotype.

How does Grindr think that a system like this could possibly work? People are worried about being rejected and stereotyped. Do we really need to portray ourselves in that way? (Paraphrased comment from NW12)

However, not all users viewed this as a negative consequence of disclosure, with some identifying a stigma avoiding benefit. If they were to disclose their HIV positive status on their public profile, users who were uninterested in sexual contact with them could organically filter them out. The following comment from NW4 describes how he used the in-built block function to block users who are HIV positive. Whilst there was a general sense in the data that users are entitled to make their own private sexual risk decisions, comments like this were often stigmatising in nature.

Being HIV Negative is better. I would never ever have sex with someone HIV positive. I just block anyone who is + so I don't have to communicate to those people who made bad decisions (Original comment from NW4)

Some comments also suggested that public HIV status disclosure could provide HIV positive users with the means to make their own evaluations of users prior to engaging with them. For instance, some described being able to use this information to filter out HIV negative users, helping them avoid HIV based rejection.

I'm HIV+ and undetectable and I'd love to be able to have the option to look specifically for other HIV+ guys. I probably wouldn't use it all the time, but sometimes it's nice to look for someone knowing that you won't be rejected out of hand for having HIV (Original comment from NW1) 
Finally, some users described how public HIV disclosure could help them normalise HIV; a longterm stigma reduction strategy. Some users felt that openness about a HIV positive status would help raise awareness, educate others, and enable the familiarity of seeing HIV positive users to reduce feelings of exceptionalism around HIV.

I think it's a right step in the direction for better public health awareness. HIV has always been stigmatized, but diagnoses are becoming more accepted with medical advances. Being positive is becoming more streamlined, but that doesn't happen if people don't talk about it (Original comment from NW4)

\subsection{User Trust}

The third theme that emerged from the data relates to trust in HIV status information being disclosed. Some comments raised concerns over the reliability of reported HIV status information due to users being uninformed, unaware, or deliberately misreporting their status to avoid being stigmatised. Trust in information disclosed due to uninformed users seems to centre around the field that allows users to disclose the date of their last HIV test. Some users reported concern at the number of reported last test dates they observed, which they considered to be out of date. Our findings suggest that reporting an out-of-date last date test could potentially devalue its corresponding HIV status, and result in undesirable signals that the user is not looking after their own sexual health. In these instances, out-of-date information appeared to act similarly to non-disclosures, causing privacy to unravel around both the last test date and reported HIV status, with stigmatising assumptions developing as a consequence of these out-of-date tests.

My other issue is the serious lack of information in the community. On a lot of profiles I see "tested negative * six months ago *" which seems to indicate these men think one test and they're good to go for long periods without retesting or don't care to (Original comment from NW1)

Men who are unaware of their HIV positive status was of particular concern to some. It was understood that by introducing HIV status information into Grindr, some men who were HIV positive but unaware might still be reporting to be HIV negative. One comment from NW8 expressed the view that this could "lead to a false sense of security" developing for people who overly rely on the information they see in Grindr and are less willing to discuss HIV in more intimate interactions. As reported above, some suggested evaluating the last test date alongside a user's declared status to assess the validity of the information, with the validity reducing as the elapsed time since the last test date increases.

that's a completely different situation from a guy who tested negative 6 months ago, and is actually telling the truth about that... but in 6 months he's barebacked 25 times and got infected and has a viral load of 300 trillion or so. THAT'S the guy you really need to worry about! (Original comment from NW6)

As our findings suggest, some HIV positive users felt stigmatised because of their status. These feelings of stigma could result in users misreporting their status as HIV negative, or negative on PrEP to avoid the stigma associated with a non-disclosure. In the longer term, this could have a negative impact on trust within the environment. Whilst Grindr provides users with a nondisclosure option, in the next section we present finding which suggests why some users may still act to misreport their status, rather than using this non-disclosure option.

As long as society continues to put a stigma on HIV, People will continue to be less honest about [their status]. Why would someone tell you the truth, when you are going to be 
mistreated. Lying about [status] isn't right but people mistreating or ostracized someone because [of] it isn't right either (Original comment from NW6)

\subsection{Providing Disclosure Choice}

Control over access and flow of personal information online is a well-established factor affecting privacy concerns when interacting online [37, 67]. For Grindr users, an important element of control is disclosure choice over when and to whom information is disclosed; especially when that information is sensitive and potentially stigmatising. As our previously reported findings suggest, the stigma around HIV could lead some users to purposefully misreport their HIV status to avoid exposure to stigma. This is reflected in our findings, where users report their desire for HIV disclosure choice. In an environment where all users are expected to disclose, privacy unraveling around non-disclosures may limit this choice.

When all said and done, it's forced disclosure that I dislike, or the fact that HIV+ users are expected to self-disclose their status straight away. Why should they? (Paraphrased comment from NW8)

A number of comments identified the optional nature of the HIV disclosure field in Grindr, with a user from NW4 stating that: "It's an optional field that isn't harming anyone". However, the privacy sensitivities around HIV status differ between user groups, with higher sensitivities and disclosure costs associated with users disclosing a HIV positive status compared to those disclosing a HIV negative status. We identified support for this, with one person reflecting on the disclosure behaviours of Grindr users in his area. He described people disclosing their HIV negative status and last test date as a means of showing off to other users, whilst other comments show HIV positive users fearing stigma.

In my area, there seems to be a rush to show (off) your hiv negative status with the date and everything and I just don't like it. It's like giving yourself a pat on the back for being lucky or "better" than other people (Original comment from NW4)

In an environment where users act to increase their own desirability, the potential for privacy to unravel around users who choose not to disclose was discussed. Identifying the unequal sensitivities around this information, several comments raised concern that stigmatising signals could develop around non-disclosing users. It was mentioned by some that this could negatively impact on their right to choose, and their right to privacy.

Putting this option on a profile is prejudicial to those who are HIV positive but do not want to declare it publicly. By allowing users to state if they are negative or positive makes it seem that, if not completed, the user is actually positive. This option is, by default, against the private rights of those with HIV. (Original comment from PF)

However, these social assumptions were not universal, with some contrasting views also present. One comment from NW1 stated that enough "alternative possibilities" existed to stop people from drawing negative conclusions, whilst another user felt non-disclosure would simply indicate that the person had decided not to disclose.

I don't think that not posting one's status means he's positive. It just means the guy don't say nothing about his status. (Original comment from PF)

Privacy, and the right to choose is an important aspect of disclosure in any online environment. This is especially true within this context as it enabled people to manage their own concerns. For some, choosing not to disclose could be a way of avoiding stigma, while for others stigma could be avoided through disclosure. Either way, if disclosure choice is removed, privacy of the user is impacted which could have a negative impact on levels of trust around HIV information online. 


\section{DISCUSSION}

The goal of this study was to explore user views of an HIV intervention integrated into a geosocial hookup app, and its potential to impact on user privacy. In this section, we first discuss some of the contrasting views identified in our analysis. We then explore the social impact of introducing HIV status information into an online geosocial hookup environment which reveals why some users may develop privacy concerns. In exploring the social impact, we identify a potential social privacy problem which could result in assumptions developing around users who choose not to disclose. Finally, using four previously identified limits to privacy unraveling [50], we propose a number of descriptive conceptual designs to provide a first look at how these limits could be applied in design within this context.

\subsection{Understanding Users}

Our analysis identified three main groups with varying views related to this HIV intervention:

(1) The first group which we refer to here as the privacy group was concerned that the public disclosure of their HIV status information could lead to increased exposure to stigma, or that Grindr was an inappropriate environment to disclose such information. Our findings are consistent with previous studies which report fear of rejection $[19,68]$ and privacy concerns related to HIV stigma $[1,30,56]$ as being reasons to withhold the disclosure of their HIV status information. We found this to be particularly pertinent in the case of public disclosure where much less control over the dissemination of the information is afforded to the individual. As such, this group indicated a preference for one-to-one HIV related discussions to increase disclosure control. (2) The second group consisted of HIV positive users who identified a potential benefit to publicly disclosing their HIV status to others as it provided them with a means of reducing their stigma exposure. When contacting and being contacted by other users, they expressed uncertainty over how their HIV status would be perceived. Supporting previous research [18], we found this group utilising public disclosure to reduce this uncertainty, allowing them to organically filter and be filtered out by individuals with whom they were at greater risk of HIV related stigma and rejection. We also found support for previous research $[20,47]$ that some users within this group disclose publicly to help them reduce HIV related stigma and to normalise HIV through a more open disclosure approach (3) Finally, some HIV negative users who were concerned about being infected with HIV viewed the publicly disclosed HIV status of others as a way of avoiding contact with HIV positive individuals, with the aim of lowering their risk of infection [68].

\subsection{Limiting Social Interactions through Public Disclosures}

Of the three main groups identified, two groups suggested public disclosure of their HIV status could reduce their stigma exposure or reduce their perceived sexual risk. The privacy group reported a preference for restricting this information to one-to-one interactions. Whilst the focus of this study was on public HIV disclosure using this explicit HIV disclosure field, we are currently conducting a follow-up study to explore disclosure strategies which include these one-to-one interactions. In this section, we aim to understand the potential impact of failing to respect the views of this privacy group and explore the potential limitations they face when interacting in this environment if their privacy is violated.

When privacy is understood as a functional requirement for identity management, associating sensitive, potentially stigmatising information to an existing online identity could cause concerns that identity will become disrupted. When interacting in any online social environment, uncertainty exists over how information being disclosed about the self will be perceived by others and whether that information will be managed appropriately [49]. For example, in Emlet's [22] interview study 
with HIV positive individuals, they found $25 \%$ of their participants had the confidentiality of their HIV status violated by others at some point.

Uncertainty over the functionality of online social environments, the social norms present, and the users operating in these environments is likely to reduce through observational learning $[6,69]$. These interactions could help users develop confidence in their environment and interactions prior to disclosure. As an example, the immediacy with which someone requests personal information and the way they ask, is feedback which may help them in forming opinions and trust. This is reduced when individual disclosure choice is removed. This can be especially pertinent in the context of HIV, with our findings supporting previous research which shows users being concerned about the social stigma HIV creates, and the rejection it can cause [19, 68]. A study found recently diagnosed MSM experiencing higher rates of mental illness in the 12 months post diagnosis [60]. As such, research has identified the need to support these individuals by fostering positive interactions during this period [62].

Respecting the privacy of this group would provide them with more control and choice over when and who they disclose to. However, disclosure choice is not limited to when, who or even if information is revealed, but also how the information is relayed. Disclosing an HIV positive status often perceived as a socially undesirable attribute - is not consistent with people's initial interaction goals of maximising social desirability [28]. Gradual, mutual self-disclosures can help develop trust between users, reducing uncertainty over how the other person may respond to new information Self-disclosing within an emotionally constructed, contextualised narrative allows users to better manage the impressions they give-off. Public disclosures - often void of these narratives - may lead the information receiver to develop their own narrative and, where a person holds out-of-date or even stigmatising views of HIV, the narrative they develop may align with those views, and increase their risk of rejection. Our findings suggest HIV positive respondents could mitigate this by disclosing later on in the interaction in more intimate one-to-one conversations (private chats) This could increase disclosure control, and allow them to shape their own narrative. This may also provide an opportunity to educate those with less knowledge and awareness of HIV. However, if these users feel unable to keep this information private using the options currently available, this form of disclosure choice becomes limited.

When disclosure choice is limited, users may develop other strategies to keep their information private. As our findings and previous literature have shown [14], the fear of rejection individuals face as a result of HIV related stigma may lead users to misreport their HIV status. Whilst introducing this information into Grindr and similar environments could have a positive impact, it is important that information being disclosed is reliable. If users who feel unable to disclose are limited in their non-disclosure choice, this has the potential to increase HIV status misreporting. For this reason, we focus on non-disclosure choice and explore the potential impact privacy unraveling can have, and ways in which this effect can be reduced.

\section{3 "Unraveling" HIV Non-Disclosures}

The privacy group reported being concerned at the public nature in which their HIV status would be available. As discussed, publicly disclosing sensitive information can limit a person's ability to manage their identity and could inhibit aspects of social interaction. To avoid this, the privacy group have the option to select 'Do not show', allowing them to keep their HIV status private. However, consistent with previous findings [52], the unequal costs to disclosure between HIV positive and negative states have the potential to cause social assumptions to develop, creating stigmatising signals. We identify this as the privacy unraveling effect [50] occurring in an online social environment where individuals are utilising signals (through disclosure) to maintain their social desirability. The Universal Design principle of Equitable Use states that a system should be 
designed in such a way that it neither disadvantages nor stigmatises any group of users [9]. The unequal sensitivity of HIV status information across users mean that requesting users to publicly disclose using this current design could violate this principle and limit the reality of disclosure choice for users wishing to keep their status undisclosed.

The appropriateness of sharing sensitive and often stigmatising health data in an online environment like Grindr was questioned in some comments. Contextual inappropriateness, as well as stigma associated with HIV could affect HIV positive and negative users alike, both of whom may prefer not to disclose their status publicly. Our findings suggest users may feel increased pressure to disclose their status through fear of non-disclosure creating stigmatising signals. Unless positive users misreport their status as being either negative, or perhaps even negative on PrEP, the privacy unraveling effect could result in stigma being attached similarly to both disclosed and non-disclosed states. Similarly with increased usage of PrEP and the stigma associated with the preventative drug $[29,38]$, users may feel pressured to disclose where non-disclosure attracts stigma. However, it is important to note that this unraveling effect may differ across cultures. As our data was collected from English language, western media outlets, it is likely to have a cultural bias. A future study exploring the cultural impact of these effects will be conducted.

\subsection{Designing out Privacy Unraveling}

In this section of the paper, we draw on Peppet's [50] four limits to privacy unraveling to propose a set of descriptive conceptual public HIV status disclosure designs. We use these conceptual designs to explore the potential benefits and restraints of using these unraveling limits in this context, to explore more detailed segregation of the limits, and to stimulate further research and discussion around privacy unraveling within the privacy and social computing communities. The conceptual design artefacts developed are not intended to change the outcome of the intervention, but to increase the privacy it affords users who prefer to keep their HIV status information undisclosed. As such, we focus the design of these artefacts on marginalised groups who experience higher risk of social stigma.

5.4.1 Transaction Cost. The first limit of privacy unraveling occurs when the cost of disclosing is increased, limiting the signal created through non-disclosure. In effect, increasing transactional cost for HIV negative users is intended to artificially re-balance the cost of HIV status disclosure. We propose a more detailed segregation of transaction cost than Peppet, proposing two subcategories (financial and time) to artificially inflate transaction cost. In making the disclosure of a desirable attribute more difficult or costly, other users may assume non-disclosure is a result of these associated disclosure costs, rather than inferring an undesirable attribute.

To increase the financial cost of disclosing HIV status information, disclosure could be limited to "premium" users who pay for using the app. Most dating applications, including Grindr, provide users with the ability to pay for membership to remove adverts and increase functionality. The aim of this design is to reduce the stigma signal associated with non-disclosure of HIV status by associating a financial cost with disclosing. However, restricting disclosure to paid users would also limit the numbers disclosing and engaging with the intervention which could reduce the intervention's overall effectiveness. Additionally, if a sufficient portion of users utilise the premium service to disclose a negative HIV status, this could stigmatise and, thus, disadvantage people who are unwilling or unable to pay for premium features.

Alternatively, we suggest a time transactional cost approach by implementing a pre-disclosure friction that requires the user's attention and interaction. Our findings in section 4.2 suggest a requirement exists to educate and raise awareness around HIV within this environment, to help reduce HIV related stigma. Therefore, we propose integrating an educational element within the 
HIV disclosure process. For example, if a user decided to disclose their HIV status, they could be required to watch an educational video or to scroll through a series of educational screens or quizzes prior to disclosing. This could act as a transactional time cost that could both educate and limit the privacy unraveling effect. Unlike the financial solution, this does not limit the disclosure function to a sub-set of users, but instead serves as an educational and HIV awareness feature. For the two groups who choose to disclose, this design would not inhibit them in doing so except for the slight increase cost in time required.

5.4.2 Unverifiability of Ignorance. Another method of limiting unraveling is to make it more difficult for the signal receiver to verify that the concealing party (signal sender) is aware of the state of their own attribute, which in this case is their HIV status. For example, Alex would be unable to unravel Bob's HIV status if Alex was unable to verify that Bob knew his own status. One method of implementing this into design would be to introduce an explicit 'I don't know' HIV status option, designed to create uncertainty over the awareness the user has of their own status. There are however a number of problems with this approach. Firstly, if a user publicly states that they are unaware of their own HIV status, our findings in section 4.3 show how this could create an undesirable signal that they are not taking care of their sexual health. Secondly, if used by an HIV positive user, it would be an inaccurate statement.

An alternative approach again draws on time, but in this design it is used to artificially increase unverifiability of ignorance. In our findings in section 4.3 we found trust in a reported HIV negative status can reduce when the last test date has elapsed beyond a certain point. Therefore, this approach implements an idle period against a person's HIV negative status so a person's status would change independently to "undisclosed" after a certain amount of time, e.g. 6 months (in line with existing HIV testing guidance). This would allow users who do not want to disclose their status to disguise themselves as people who haven't spotted that their profile idled back to the undisclosed state. For HIV negative users, it would also promote a periodic sexual health check. For HIV positive users who choose to disclose, their status would not need to be reset and could remain static to avoid having to repeat this unnecessary disclosure step. Whilst this could lead to stigma for users who do not keep their profile up-to-date, this stigma would only be likely if the majority of other users frequently updated their profile information.

5.4.3 Inability to Accurately Infer the Negative. The third limitation occurs when it is not possible to accurately infer an undesirable attribute through non-disclosure. Previous attempts have been made to introduce ambiguity into the HIV disclosure process which could help reduce users' ability to infer undesirable attributes. One real-time MSM dating website requires all of their users to disclose their HIV status, but provides an optional "Ask Me" flag to limit disclosure to others [34] This removes the non-disclosure state but replaces it with an "Ask Me" state. Whilst this does set itself apart from the more traditional approaches of providing a non-disclosure option, a state still exists around which undesirable assumptions can be inferred. Drawing on this approach, our design concept uses similar ambiguities, but includes data clustering to remove these single non-disclosure states. An example of this would be information grouping, allowing users to mark a group of fields as undisclosed rather than each individually. It is important to note here that HIV status information is not the only information type that is subject to social stigma within these environments. Age, relationship status, ethnicity, height and other information types can be a cause of stigma [33], or even stigma upon stigma (e.g. an older HIV positive man may be stigmatised for his age as well as his HIV status). Some of these information types could be approximately inferred from a user's profile picture if their facial features are visible (e.g. age, ethnicity), whereas others such as HIV status cannot. This design could create uncertainty over all the explicit fields the user is unwilling to disclose publicly which cannot otherwise be inferred, reducing the accuracy around inferences 
made, whilst at the same time allowing disclosure of information for those who choose to make it public.

5.4.4 Norms. The final limit of privacy unraveling can occur when norms develop around nondisclosures. Previous research identified disclosure norms which have developed around the exchange of pictures in MSM hookup apps, with some conversations being dependent on the exchange of personal pictures (e.g. "no pic no chat") and certain requirements being applied to these pictures (e.g. "no headless torsos") [63]. However, around HIV status information, apps like Grindr could encourage norms to develop within their environments by removing the option to disclose an HIV negative status, e.g. providing disclosure options for every status other than HIV negative and negative on PrEP, effectively allowing only positive users to disclose. The benefit of this approach would be a limitation of the unraveling effect, as stigmatising assumptions would be much less likely to develop around non-disclosure when not all disclosure options are available within the explicit field. However, removing the option for HIV negative users would limit engagement with the intervention, and may further isolate HIV positive users who would be singled out when disclosing their status. Alternative HIV reporting models have been introduced in other MSM dating environments that move away from explicit HIV negative status disclosure. The dating app Scruff for example does not provide users the option to disclose HIV status information, instead it asks users to disclose their safer sex practices i.e. Condoms, PrEP, Treatment as Prevention (TasP). Interestingly they also build ambiguity into their design by not explicitly stating whether these safer sex practices are ones the user is adhering to, or practices they are looking for in others. This approach however would disadvantage HIV positive users who disclose publicly to avoid HIV related rejection, as well as HIV negative users wanting to be open about their status and aware of the status of others.

5.4.5 Cultural Signals. Whilst not a limit of unraveling, designers could also consider ways to reduce the stigmatising costs of disclosing a HIV positive status within these types of geosocial hookup applications. Previous efforts have been made to attempt culture changes in MSM dating environments by asking users to pledge to live "stigma free lives" [42]. Whilst this would not limit the unraveling effect around non-disclosures, it might reduce the cost of unraveling if it occurred, through reduced social stigma associated to the undesirable attribute.

\section{LIMITATIONS}

The use of online comments has limitations. For instance, these findings are based on self-reported behaviours and motivations which may not translate into actual in situ behaviours. Using this data source removes the ability to direct the conversation, limiting the scope to the discussions raised by participants. Participants are also self-selecting, and could consist of a group who are more dominant in certain discussions, leaving the voices of the broader audience to remain comparatively quiet, homogenising the debate [3]. The often anonymous nature of these spaces make it difficult to ascertain demographic information, and as our study used comments from English language, western news outlets, certain demographics are likely to be underrepresented. The comments are also limited to details those commenting feel comfortable disclosing in an online public space which should be given special recognition when conducting research around a sensitive topic. However, the anonymous nature of these online spaces may provide an environment where individuals feel more able to discuss certain topics, although this anonymity may also lower the quality of the discourse [35].

As such, studies using online comments as a data source are not necessarily generalisable to the wider population, although they may generalise to a smaller segment of the population [36], and so to help support our findings, where possible we evaluate them with previous research. We also 
suggest further work presented in section 7 which could be conducted to externally validate our claims.

\section{FURTHER WORK}

In conducting this research, we have identified several areas of further research which could help develop our understanding of privacy unraveling within the privacy and social computing fields. Firstly, we suggest the development of a quantitative measure for evaluating the level of privacy unraveling that exists around sensitive data in online social environments. This would allow future researchers to evaluate unraveling reducing designs such as those proposed in this paper. Using this measure, we recommend that HIV disclosure designs in existing MSM dating applications be evaluated alongside alternative prototype designs, allowing us to further investigate unraveling limitations. We are conducting an in-depth interview study with HIV positive and negative users to understand the implications of the unraveling effect in greater depth. This follow-up study will provide a better understanding of how users develop strategies around signals that develop within these online environments. Finally, we propose an observation study to explore the external validity of our findings which, currently rely on self-reported data.

\section{CONCLUSIONS}

In this paper, we present the findings from an online qualitative analysis of online comments about an HIV intervention that was integrated into the online geosocial hookup app Grindr. Our analysis identified privacy concerns when linking sensitive health information to an existing online identity due to the stigma that HIV attracts. We found this stigma can create a barrier to disclosure, with some users choosing to keep their HIV status information private to reduce their stigma exposure, which supports previous research. In addition to this, we also found that fear of being stigmatised could cause some men to publicly disclose to allow them to organically filter out, or be filtered out by users from whom they were at greater risk of HIV related stigma and rejection. Where users preferred to keep their HIV status information private, we identified a potential limit to the non-disclosure options that Grindr provides. We found that the HIV status of users who choose not to disclose can sometimes be inferred through their non-disclosure decision, an effect known as privacy unraveling. To address this, we reviewed four methods that can limit this effect [50], for which we developed a number of sub-categories and descriptive conceptual designs to explore the potential for these limits to be applied in design within this context. By doing so, we extend the literature on privacy unraveling and identify a new application domain within online social environments.

\section{ACKNOWLEDGMENTS}

This work is partially funded by the EU Horizon 2020 research and innovation program under the Marie Skłodowska-Curie Action ITN grant agreement No 675730 and the University College London (UCL) Department of Computer Science.

\section{REFERENCES}

[1] Barry D. Adam, James Murray, Suzanne Ross, Jason Oliver, Stephen G. Lincoln, and Vicki Rynard. 2011. Hivstigma.com, an innovative web-supported stigma reduction intervention for gay and bisexual men. Health Education Research 26, 5 (2011), 795-807. https://doi.org/10.1093/her/cyq078

[2] Ann Adams and M. Angela Sasse. 2001. Privacy in multimedia communications: Protecting users, not just data. In People and Computers XV-Interaction without Frontiers (2001), 49-64. http://link.springer.com/chapter/10.1007/ 978-1-4471-0353-0_4

[3] Steffen Albrecht. 2006. Whose voice is heard in online deliberation?: A study of participation and representation in political debates on the internet. Information, Community and Society 9, 1 (2006), 62-82. 
[4] Nazanin Andalibi and Oliver L Haimson. 2016. Understanding Social Media Disclosure s of Sexual Abuse Through the Lens es of Support Seeking and Anonymity. In Proceedings of the 2016 CHI Conference on Human Factors in Computing Systems. ACM.

[5] Andrew Anglemyer, George W Rutherford, Rachel C Baggaley, Matthias Egger, and Nandi Siegfried. 2011. Antiretroviral therapy for prevention of HIV transmission in HIV-discordant couples. FAMA 310, 15 (2011), 1619-1620. https: //doi.org/10.1002/14651858.CD009153.pub2

[6] Tamar Ashuri, Shira Dvir-Gvisman, and Ruth Halperin. 2018. Watching Me Watching You: How Observational Learning Affects Self-disclosure on Social Network Sites? Journal of Computer-Mediated Communication 23, 1 (2018), 34-68. https://doi.org/10.1093/jcmc/zmx003

[7] Lisa M. Baumgartner. 2007. The Incorporation of the HIV/AIDS Identity Into the Self Over Time. Qualitative Health Research 17, 7 (2007), 919-931. https://doi.org/10.1177/1049732307305881

[8] Volker Benndorf, Dorothea Kübler, and Hans Theo Normann. 2015. Privacy concerns, voluntary disclosure of information, and unraveling: An experiment. European Economic Review 75 (2015), 43-59. https://doi.org/10.1016/j euroecorev.2015.01.005

[9] David Benyon. 2014. Designing Interactive Systems: A comprehensive guide to HCI, UX and interaction design, 3/E.

[10] Daniela Bezemer, Frank de Wolf, Maarten C Boerlijst, Ard van Sighem, T Deirdre Hollingsworth, Maria Prins, Ronald B Geskus, Luuk Gras, Roel A Coutinho, and Christophe Fraser. 2008. A resurgent HIV-1 epidemic among men who have sex with men in the era of potent antiretroviral therapy. Aids 22, 9 (2008), 1071-1077. https://doi.org/10.1097/QAD 0b013e $3282 \mathrm{fd} 167 \mathrm{c}$

[11] Jeremy Birnholtz, Colin Fitzpatrick, Mark Handel, and Jed R Brubaker. 2014. Identity, Identification and Identifiability: The Language of Self-Presentation on a Location-Based Mobile Dating App. To Appear in Proc. MobileHCI 2014 (2014), 3-12. https://doi.org/10.1145/2628363.2628406

[12] Virginia Braun and Victoria Clarke. 2008. Using thematic analysis in psychology. Qualitative Research in Psychology 3 , October 2012 (2008), 37-41. https://doi.org/10.1191/1478088706qp063oa

[13] AE Brown, S Nash, N Connor, PD Kirwan, D Ogaz, S Croxford, D De Angelis, and VC Delpech. 2017. Towards elimination of HIV transmission, AIDS and HIV-related deaths in the UK. HIV medicine (2017).

[14] Alex Carballo-Diéguez, Michael Miner, Curtis Dolezal, B. R Simon Rosser, and Scott Jacoby. 2006. Sexual negotiation, HIV-status disclosure, and sexual risk behavior among latino men who use the internet to seek sex with other men. Archives of Sexual Behavior 35, 4 (2006), 473-481. https://doi.org/10.1007/s10508-006-9078-7

[15] Stephenie R. Chaudoir and Jeffrey D Fisher. 2010. The disclosure processes model: Understanding disclosure decision making and postdisclosure outcomes among people living with a concealable stigmatized identity. Psychological Bulletin 136, 2 (2010), 236-256. https://doi.org/10.1037/a0018193

[16] Jeremy Y Chow and Jeffrey D Klausner. 2018. Use of geosocial networking applications to reach men who have sex with men: progress and opportunities for improvement.

[17] JD Davids. 2016. Grindr, Largest Gay Hookup App, Adds Fields for HIV Status, Undetectable, and PrEP Use. http: //www.thebody.com/content/78739/grindr-largest-gay-hookup-app-adds-fields-for-hiv-.html

[18] Mark Davis, Graham Hart, Graham Bolding, Lorraine Sherr, and Jonathan Elford. 2006. E-dating, identity and HIV prevention: Theorising sexualities, risk and network society. Sociology of Health and Illness 28, 4 (2006), 457-478. https://doi.org/10.1111/j.1467-9566.2006.00501.x

[19] Valerian Derlega, Kathryn Green, Julianne Serovich, and William Elwood. 2002. Perceived HIV-related Stigma and HIV Disclosure to Relationship Partners after Finding Out about the Seropositive Diagnosis. Fournal of Health Psychology 7 , 4 (2002), 415-432.

[20] Valerian J Derlega, Barbara A Winstead, Kathryn Greene, Julianne Serovich, and William N Elwood. 2004. Reasons for HIV disclosure/nondisclosure in close relationships: Testing a model of HIV-disclosure decision making. Fournal of Social and Clinical Psychology 23, 6 (2004), 747-767.

[21] Deborah Donnell, Jared M. Baeten, James Kiarie, Katherine K. Thomas, Wendy Stevens, Craig R. Cohen, James McIntyre, Jairam R. Lingappa, and Connie Celum. 2010. Heterosexual HIV-1 transmission after initiation of antiretroviral therapy: a prospective cohort analysis. The Lancet 375, 9731 (2010), 2092-2098. https://doi.org/10.1016/S0140-6736(10)60705-2

[22] C a Emlet. 2008. Truth and consequences: a qualitative exploration of HIV disclosure in older adults. AIDS care 20, 6 (2008), 710-7. https://doi.org/10.1080/09540120701694014

[23] PH England. 2015. HIV in the UK-Situation Report 2015 Incidence, prevalence and prevention. Prot. Improv. Nations Heal 1, 1 (2015), 1-20.

[24] Casey Fiesler and Blake Hallinan. 2018. "We Are the Product": Public Reactions to Online Data Sharing and Privacy Controversies in the Media. Chi 2018 (2018), 13. https://doi.org/10.1145/3173574.3173627

[25] Paul Flowers, Mark McGregor Davis, Michael Larkin, Stephanie Church, and Claire Marriott. 2011. Understanding the impact of HIV diagnosis amongst gay men in Scotland: An interpretative phenomenological analysis. Psychology \& Health 26, 10 (2011), 1378-1391. https://doi.org/10.1080/08870446.2010.551213 
[26] Azeen Ghorayshi and Sri Ray. [n. d.]. Grindr Is Letting Other Companies See User HIV Status And Location Data. https://www.buzzfeed.com/azeenghorayshi/grindr-hiv-status-privacy?utm\{_\}term=.xorXWLx2\{\#\}.mdk3Q5ml

[27] N M Glenn, C C Champion, and J C Spence. 2012. Qualitative content analysis of online news media coverage of weight loss surgery and related reader comments. Clinical obesity 2, 5 (2012), 125. https://doi.org/10.1111/cob.12000

[28] Erving Goffman. 1956. The presentation of self in everyday life. Vol. 21. xii, 259 p. pages. https://doi.org/10.2307/258197

[29] Sarit A. Golub. 2018. PrEP Stigma: Implicit and Explicit Drivers of Disparity. Current HIV/AIDS Reports 15, 2 (2018), 190-197. https://doi.org/10.1007/s11904-018-0385-0

[30] K Greene, VJ Derlega, GA Yep, and S Petronio. 2003. Privacy and disclosure of HIV in interpersonal relationships: A sourcebook for researchers and practitioners.

[31] Grindr. 2017. Grindr Factsheet 2017. https://www.grindr.com/press/

[32] Irene Hall, David Holtgrave, and Catherine Maulsby. 2012. HIV transmission rates from persons living with HIV who are aware and unaware of their infection, United States. AIDS (London, England) (2012), 8-10. https://doi.org/10.1097/ QAD.0b013e328351f73f

[33] Chong-suk Han. 2008. No fats, femmes, or Asians: the utility of critical race theory in examining the role of gay stock stories in the marginalization of gay Asian men. Contemporary Justice Review 11, 1 (2008), 11-22. https: //doi.org/10.1080/10282580701850355

[34] Mark J. Handel and Irina Shklovski. 2012. Disclosure, ambiguity and risk reduction in real-time dating sites. Proceedings of the 17th ACM international conference on Supporting group work - GROUP '12 (2012), 175. https://doi.org/10.1145/ 2389176.2389203

[35] Eszter Hargittai and Amanda Hinnant. 2008. Differences in Young Adults ' Use of the Internet. Communication Research 35, 5 (2008), 602-621. https://doi.org/10.1177/0093650208321782

[36] Natalie Henrich and Bev Holmes. 2013. Web news readers' comments: Towards developing a methodology for using on-line comments in social inquiry. Fournal of Media and Communication Studies 5, 1 (2013), 1-4. https: //doi.org/10.5897/JMCS11.103

[37] Christopher M. Hoadley, Heng Xu, Joey J. Lee, and Mary Beth Rosson. 2010. Privacy as information access and illusory control: The case of the Facebook News Feed privacy outcry. Electronic Commerce Research and Applications 9, 1 (2010), 50-60. https://doi.org/10.1016/j.elerap.2009.05.001

[38] Rusi Jaspal and Christos Daramilas. 2016. Perceptions of pre-exposure prophylaxis (PrEP) among HIV-negative and HIV-positive men who have sex with men (MSM). Cogent Medicine 3, 1 (2016), 1-16. https://doi.org/10.1080/2331205X 2016.1256850

[39] Adam N. Joinson and Carina B. Paine. 2007. Self-disclosure, Privacy and the Internet. Oxford Handbook of Internet Psychology (2007), 237-252. https://doi.org/10.1093/oxfordhb/9780199561803.013.0016

[40] Linnea I Laestadius and Mark A Caldwell. 2015. Is the future of meat palatable? Perceptions of in vitro meat as evidenced by online news comments. Public Health Nutrition 18, 13 (2015), 1-11. https://doi.org/10.1017/S1368980015000622

[41] Mark R. Leary. [n. d.]. Self-presentation: Impression management and interpersonal behavior. 246-xv, 246 pages. https://doi.org/im

[42] Karen Levy and Solon Barocas. 2017. Designing against discrimination in online markets. Berkeley Technology Law fournal 32 (2017). https://ssrn.com/abstract=3084502

[43] Gregory Lewis. 2011. Asymmetric information, adverse selection and online disclosure: The case of ebay motors. American Economic Review 101, 4 (2011), 1535-1546. https://doi.org/10.1257/aer.101.4.1535

[44] Juan F Maestre, Haley Macleod, Ciabhan L Connelly, Julia C Dunbar, Jordan Beck, Katie A Siek, and Patrick C Shih. 2018. Defining Through Expansion : Conducting Asynchronous Remote Communities ( ARC) Research with Stigmatized Groups. In Proc. of CHI (2018), 1-13. https://doi.org/10.1145/3173574.3174131

[45] Sarah McRoberts, Haiwei Ma, Andrew Hall, and Svetlana Yarosh. 2017. Share First, Save Later: Performance of Self through Snapchat Stories. In Proceedings of the 2017 CHI Conference on Human Factors in Computing Systems - CHI '17. 6902-6911. https://doi.org/10.1145/3025453.3025771

[46] Kathryn E. Muessig, Emily C. Pike, Sara Legrand, and Lisa B. Hightow-Weidman. 2013. Mobile phone applications for the care and prevention of HIV and other sexually transmitted diseases: A review. fournal of Medical Internet Research 15, 1 (2013), 1-29. https://doi.org/10.2196/jmir.2301

[47] P. J. Murphy, D. Hevey, S. O’Dea, N. Ni Rathaille, and F. Mulcahy. 2015. Serostatus Disclosure, Stigma Resistance, and Identity Management Among HIV-Positive Gay Men in Ireland. Qualitative Health Research (2015), 1049732315606687. https://doi.org/10.1177/1049732315606687

[48] NHS. 2018. HIV and AIDS. https://www.nhs.uk/conditions/hiv-and-aids/prevention/

[49] Helen Nissenbaum. 2009. Privacy in context: Technology, policy, and the integrity of social life. Stanford University Press.

[50] Scott R Peppet. 2011. Unraveling privacy: The personal prospectus and the threat of a full-disclosure future. Nw. UL Rev. 105 (2011), 1153.

Proceedings of the ACM on Human-Computer Interaction, Vol. 2, No. CSCW, Article . Publication date: November 2018. 
[51] Andrew N. Phillips, Valentina Cambiano, Fumiyo Nakagawa, Alison E. Brown, Fiona Lampe, Alison Rodger, Alec Miners, Jonathan Elford, Graham Hart, Anne M. Johnson, Jens Lundgren, and Valerie C. Delpech. 2013. Increased HIV Incidence in Men Who Have Sex with Men Despite High Levels of ART-Induced Viral Suppression: Analysis of an Extensively Documented Epidemic. PLoS ONE 8, 2 (2013), 15-16. https://doi.org/10.1371/journal.pone.0055312

[52] Jorge Ramallo, Thomas Kidder, Tashuna Albritton, Gary Blick, John Pachankis, Valen Grandeleski, and Trace Kershaw. 2015. Exploring social networking technologies as tools for hiv prevention for men who have sex with men. AIDS Education and Prevention 27, 4 (2015), 298-311. https://doi.org/10.1521/aeap.2015.27.4.298

[53] Eric Rice, Ian Holloway, Hailey Winetrobe, Harmony Rhoades, Anamika Barman-Adhikari, Jeremy Gibbs, Adam Carranza, David Dent, and Shannon Dunlap. 2012. Sex risk among young men who have sex with men who use Grindr, a smartphone geosocial networking application. Journal of AIDS and Clinical Research 3, SPL ISSUE4 (2012) https://doi.org/10.4172/2155-6113.S4-005

[54] Alison Rodger, Valentina Cambiano, Tina Bruun, Pietro Vernazza, Simon Collins, Olaf Degen, et al. 2018. HIV transmission risk through condomless sex in gay couples with suppressive ART: The PARTNER2 Study extended results in gay men. (2018). https://www.chip.dk/Studies/PARTNER/PARTNER-press-release-2018 AIDS2018.

[55] Alison J. Rodger, Valentina Cambiano, Tina Bruun, Pietro Vernazza, Simon Collins, Jan van Lunzen, Giulio Maria Corbelli, Vicente Estrada, Anna Maria Geretti, Apostolos Beloukas, David Asboe, Pompeyo Viciana, Félix Gutiérrez, Bonaventura Clotet, Christian Pradier, Jan Gerstoft, Rainer Weber, Katarina Westling, Gilles Wandeler, Jan M. Prins, Armin Rieger, Marcel Stoeckle, Tim Kümmerle, Teresa Bini, Adriana Ammassari, Richard Gilson, Ivanka Krznaric, Matti Ristola, Robert Zangerle, Pia Handberg, Antonio Antela, Sris Allan, Andrew N. Phillips, and Jens Lundgren. 2016. Sexual Activity Without Condoms and Risk of HIV Transmission in Serodifferent Couples When the HIV-Positive Partner Is Using Suppressive Antiretroviral Therapy. Jama 316, 2 (2016), 171. https://doi.org/10.1001/jama.2016.5148

[56] Julianne M Serovich and Katie E Mosack. 2006. Reasons for Hiv Disclosure or Nondisclosure To Casual Sexual Partners. Aids 15, 1 (2006), 70-80.

[57] Suvi Silfverberg, Lassi a Liikkanen, and Airi Lampinen. 2010. "I’ll Press Play, But I Won’t Listen": Profile Work in a Music-focused Social Network Service.. In Proceedings of the ACM 2011 conference on Computer supported cooperative work. 1-10. https://doi.org/10.1145/1958824.1958855

[58] Aneesha Singh, Joe Gibbs, Claudia S Estcourt, Pam Sonnenberg, and Ann Blandford. 2017. Are HIV Smartphone Apps and Online Interventions Fit for Purpose?. In Proceedings of the 2017 International Conference on Digital Health. ACM, 2017. London. https://doi.org/10.1145/3079452.3079469

[59] Katarzyna Stawarz, Anna L Cox, and Ann Blandford. 2014. Don't Forget Your Pill! Designing Effective Medication Reminder Apps That Support Users' Daily Routines. In Proceedings of the 32nd annual ACM conference on Human factors in computing systems. ACM, 2014.

[60] stigmaindexuk.org. 2015. The People Living with HIV Stigma Survey UK. Men Who Have Sex With Men (MSM).

[61] Samuel Hardman Taylor, Jevan Hutson, and Tyler Richard Alicea. 2017. Social Consequences of Grindr Use : Extending the Internet-Enhanced Self-Disclosure Hypothesis. In Proceedings of the 2017 CHI Conference on Human Factors in Computing Systems. ACM.

[62] Yelena Tsarenko and Michael Jay Polonsky. 2011. "You can spend your life dying or you can spend your life living": Identity transition in people who are HIV-positive. Psychology \& Health 26, 4 (2011), 465-483. https://doi.org/10.1080/ 08870440903521761

[63] Evangelos Tziallas. 2015. Gamified Eroticism: Gay Male "Social Networking" Applications and Self-Pornography. Sexuality and Culture 19, 4 (2015), 759-775. https://doi.org/10.1007/s12119-015-9288-z

[64] Chad Van De Wiele and Stephanie Tom Tong. 2014. Breaking Boundaries : The Uses \& Gratifications of Grindr. 2014 ACM International foint Conference on Pervasive and Ubiquitous Computing (2014), 619-630. https://doi.org/10.1145/ 2632048.2636070

[65] John Vines, Anja Thieme, Rob Comber, Mark Blythe, Peter Wright, and Patrick Olivier. 2013. HCI in the press: Online public reactions to mass media portrayals of HCI research. Proc. CHI 2013 (2013), 1873-1882. https://doi.org/10.1145/ 2470654.2466247

[66] Lawrence R Wheeless. 1978. A follow-up study of the relationships among trust, disclosure, and interpersonal solidarity. Human Communication Research 4, 2 (1978), 143-157.

[67] Heng Xu. 2007. The effects of self-construal and perceived control on privacy concerns. ICIS 2007 proceedings (2007), 125.

[68] María Cecilia Zea, Carol A Reisen, Paul J Poppen, and Rafael M Díaz. 2003. Asking and telling: communication about HIV status among Latino HIV-positive gay men. AIDS and Behavior 7, 2 (2003), 143-152.

[69] Douglas Zytko, Sukeshini A Grandhi, and Quentin Gad Jones. 2014. Impression management through communication in online dating. Proceedings of the companion publication of the 17th ACM conference on Computer supported cooperative work \& social computing. (2014), 277-280. https://doi.org/10.1145/2556420.2556487 
Received April 2018; revised July 2018; accepted September 2018

Proceedings of the ACM on Human-Computer Interaction, Vol. 2, No. CSCW, Article . Publication date: November 2018. 